\title{
COMERCIANTES MALLORQUINES ANTE EL ESCLAVISMO TARDÍO: ELEMENTOS PARA UN ESTUDIO
}

Antumi Toasijé

Centro de Estudios Panafricanos, Parla

RESUMEN: Apenas se han hecho intentos de cuantificar la importancia de los tratantes mallorquines en el esclavismo atlántico. Esta importancia debe ser establecida en relación con diversos elementos de análisis dispersos, cuyo estudio en profundidad puede ayudar a ofrecer un panorama más preciso. Uno de estos elementos lo constituyen las sagas familiares mallorquinas en Puerto Rico y Cuba, mientras que otro importante indicador se relaciona con la conmoción que producen en la isla las disposiciones británicas para la represión del tráfico esclavista.

Palabras clave: Trata esclavista Atlántica, Mallorca, Puerto Rico, Siglo XIX.

\section{MAJORCAN MERCHANTS FACING LATE SLAVERY: ELEMENTS FOR A STUDY}

ABSTRACT: Few attempts have been made to evaluate the importance of the involvement of the Majorcan traders in the trans-Atlantic slavery system. This importance must be established linking dispersed elements of analysis. The in deep study of these elements can help to establish a more precise picture. One of these elements are the Majorcan family sagas in Puerto Rico and Cuba, while another important indicator is related to the general commotion produced by the British dispositions for the repression of the slave trade.

Keywords: Trans-Atlantic slavery Trade, Majorca, Puerto Rico, XIX Century. 


\section{El estado actual de las investigaciones; necesidad de profundización}

Tenemos escasos estudios sobre la implicación de personas de Baleares en las diversas esferas de actividad esclavista que denominamos tardía y éstos estudios no son específicos, citaré tres: la comunicación de Joana María Escartín Bisbal al Congrés Internacional d'Estudis Històrics de 1992', en la que se transcribe una propuesta del Marqués de La Torre, al Rey de España, de datación no fijada, para establecer una compañía tratante de esclavos. En este caso nos hallamos con un documento no propiamente tardío ${ }^{2}$. Y las monografías, de A. Cubano ${ }^{3}$ y M. Segura ${ }^{4}$. Estos últimos trabajos se centran en la experiencia migratoria de familias mallorquinas haciendo escasa mención a la esclavitud como institución económica y las repercusiones reales de dicho tráfico, así como al esclavismo como ideología.

Las ponencias del Congrés Internacional d'Estudis Històrics, son una de las bases historiográficas que poseemos sobre las relaciones de Baleares y América, y contienen para el período y tema que nos interesa dos valiosísimas aportaciones; los estudios de Sebastiá Serra ${ }^{5}$ y de Antonio Manera ${ }^{6}$ sobre las trasferencias de población a las colonias españolas y territorios libres de América. De tales estudios se desprende que la emigración balear sobre todo a las Antillas Cuba y Puerto Rico reviste una importancia crucial a partir de la tercera década del diecinueve. De estos contingentes de emigrantes entre los que destacan los sollerenses, descuellan apellidos de comerciantes que llegan a amasar fortunas en las Américas iniciando auténticas sagas familiares. Pensamos que es en el relato de lo acontecido en el seno de estas familias, donde pueden hallarse, y apenas se han querido buscar, los elementos necesarios para establecer el modo de producción preferente de dichas sagas familiares y la naturaleza de las transacciones comerciales que gestionaban tales familias, así como su influencia a ambos lados del Atlántico. Obras de carácter autobiográfico adquieren así singular trascendencia, se deben tener especialmente en cuenta libros como el de Esperanza

1. Joana María Escartín Bisbal, "Sobre el tràfec de negres a la mallorca setcentista", Congrés Internacional d'Estudis Històrics, Les Illes Balears i América, Palma 1992, Coordinador Román Piña Homs.

2. Sobre aclaración del término esclavismo tardío véase en este mismo trabajo: Breves apuntes sobre el esclavismo tardío español.

3. Astrid Cubano, Un puente entre Mallorca y Puerto Rico: la emigración de Sóller (18301930) Júcar, Gijón, 1993.

4. M. Segura, Un lejano aroma de café: crónica de mallorquines en Puerto Rico. Palma de Mallorca, 1997 L. Muntaner.

5. Sebastía Serra Busquets, "La emigració de les Illes Balears a América", Congrés Internacional d'Estudis Històrics, Les Illes Balears i América, Palma 1992, Coordinador Román Piña Homs.

6. Antonio Manera Riutort, "Notes sobre les relacions comercials entre Mallorca, les coIonies de Cuba i Puerto Rico fins al 1895", Congrés Internacional d'Estudis Històrics, Les Illes Balears i América, Palma 1992, Coordinador Román Piña Homs. 
Mayol Alcover ${ }^{7}$, monografías como la de Pedro Antonio Morell Muñoz ${ }^{8}$, los artículos biográficos de Francisco C. Vicens ${ }^{9}$ y las antologías de Carlos Domínguez Cristóbal ${ }^{10}$ que hacen referencia a la tenencia de haciendas y a las actividades comerciales de los mallorquines en América. El propio Carlos Domínguez Cristobal, el más prolífico de estos autores, se concentra en las actividades de la economía esclavista en su obra: La esclavitud, la instrucción pública y la iglesia en Ciales durante el siglo XIX ${ }^{11}$.

Estela Cifre de Loubriel ${ }^{12}$ en su estudio concede toda la importancia que tuvo el componente mallorquín entre los hacendados y comerciantes de Puerto Rico, de este trabajo casi de genealogía pura deberían partir todos los que quieran hacer una historia de los mallorquines en Puerto Rico. El papel coprotagonista de los hacendados mallorquines en los hechos del Ilamado Grito de Lares, (1868 Lares, Puerto Rico), levantamiento anti-colonial y anti-esclavista que tuvo por objetivos a catalanes y mallorquines entregados a crueles formas de tortura con los esclavizados y peones queda de manifiesto en Carlos López Dzur ${ }^{13}$, historiador de la población del Pepino en Puerto Rico, el cual sigue el rastro de nombres dejado por Cifré de Loubriel para elaborar unas entrevistas de las que se obtienen importantes manifestaciones como la que reproducimos a continuación, en este caso la diferenciación entre peones y negros implica casi sin duda que los segundos son esclavos:

Los catalanes y mallorquines por ser muy abusadores con peones y negros, se ganaron la mala voluntad de mucha gente decente y también de otros agricultores ricos; nadie quería trabajarles la tierra ni darles crédito y, poquito a poco, la peonada se iba para trabajar con los Echeandía y Cabrero, antes que con Alers, Prat y Arana... Quien vino a pagarlo fue doña Lala, la última dueña de la hacienda Los Vélez. (A ella) quemaron antes que a ninguno otro, cuando la revuelta de Lares y, entonces, se hizo una apuesta para perjudicar a los catalanes y mallorquines. Le dijeron a

7. Esperanza Mayol Alcover, Islas: autobiografía, ed. Palma 1976.

8. Pedro Antonio Morell Muñoz, El Solar Morell de Camuy, sus ascendientes y descendiente, San Juan de Puerto Rico, 1995.

9. Francisco C. Vicens, "Los Mallorquines: su Contribución al Desarrollo de Ciales" en Revista Ciales Ayer y Hoy Año II, Núm. 2, 1983.

10. Carlos Dominguez Cristobal, Antología histórica de Ciales 1820-1867, Quebradillas: Imprenta San Rafael, Puerto Rico 2005, y Antología histórica de Ciales 1868-1899, Río Piedras: Jay Ce-Printing, Puerto Rico, 1994.

11. Carlos Domínguez Cristobal, La esclavitud, la instrucción pública y la iglesia en Ciales durante el siglo XIX, Editora Corripio, C. por A.; Santo Domingo, República Dominicana, 1985.

12. Estela Cifre de Loubriel, La formación del pueblo puertorriqueño: La contribución de los vascongados, navarros y aragoneses, Instituto de Cultura Puertorriqueña, 1986.

13. http://www.geocities.com/soHo/den/2060/pepino3.html. 
Vidal, Arana y Vendrell: ¡se van a tener que ir! Igual que Manuel Prat, pero él no se fue y Casildo (Vélez del Río), ése sí14

Cuba es otro foco de afluencia migratoria balear. En 1833 tenemos 8 comerciantes mallorquines asentados en Santiago de Cuba, entre 1841 y 1849 son 39 los baleares que se asientan en Santiago y entre 1833 y 1841 hay 19 comerciantes de baleares en la Habana ${ }^{15}$. La importancia del tráfico comercial con Cuba queda registrada en Joan Pou Muntaner ${ }^{16}$ que apunta ya desde 1800 un comercio con Cuba de las polacras Concepción y Príncipe de Asturias y del bergantín Sansón. A partir de 1837 empiezan para este autor los años de auge comercial con las Antillas, en 1837 parten para las Antillas las polacras Isidro, San José, Carmen, Liebre, Lealtad, San Nicolás, Trinidad, Isabel, Virgen del Carmen y San Rafael. También las goletas Palmira, Atrevida, María y Palma.

Sobre el caso, venezolano y en lo referente al cono sur nos hemos hallado con un desierto historiográfico que deberá poblarse con próximas aportaciones, debe observarse que la contribución balear con ser numerosa a partir de la segunda mitad del siglo XIX no parece serlo tanto en épocas anteriores, antes de las independencias, lo cual estrecha la horquilla temporal de la implicación en el esclavismo dadas las aboliciones americanas. Para una introducción a estas fuentes deberemos acudir de nuevo al Congrés Internacional d'Estudis Històrics.

Volviendo al caso puertorriqueño, cabe pues invitar al investigador interesado en la cuestión los esclavizados por parte de Mallorquines y otros Baleáricos en Puerto Rico, a que siga el procedimiento de cotejar los nombres recogidos por Estela Cifré17, con los censos sobre propietarios de esclavizados que están en los archivos de la isla caribeña. Un ejemplo sobre estos censos además de documentos recogidos y transcritos se encuentran en http://www.preb.com, como se muestra en el Anexo 1 (pp. 22) ${ }^{18}$.

Se debe valorar por su difusión el trabajo realizado a través de Internet de estos divulgadores de la historia de Puerto Rico, Cuba y otras zonas de América con presencia de comerciantes y hacendados mallorquines. Así el texto de Melvin Rivera Velázquez ${ }^{19}$ sobre los Alomar en Santa Isabel de Puerto Rico arroja in-

14. Carlos López Dzur, Entrevista con Antonio González Rodríguez, realizada el 16 de julio de 1978 http://www.geocities.com/soHo/den/2060/pepino3.html.

15. Ibíd. 2.

16. Joan Pou Muntaner, El Comerç Amb les Amériques Gráfiques Miramar, Palma de MaIlorca 1991.

17. Estela Cifre de Loubriel, La Formación del Pueblo Puertoriqueño.

18. La Web "PReb" (Puerto Rico en breve) http://www.preb.com presenta datos provenientes de los archivos puertoriqueños: Archivo General de Puerto Rico, Archivo Histórico de Caguas, Archivo Histórico de Mayagüez, Archivo Histórico de San Germán, Archivo Diocesano de la Arquidiócesis de San Juan y el Instituto de Estudios Históricos Alejo Arizmendi.

19. Melvin Rivera Velázquez, "Los Alomar una familia mallorquina en Santa Isabel" http://www.santaisabelpr.com/los-alomar-una-familia-mallorquina-en-santa-isabel. 
formación detallada sobre la formación y auge de esta saga esclavista con origen en la población de Sóller. No cabe duda que también a través de estos estudios avanza la comprensión sobre las implicaciones morales e ideológicas del esclavismo decimonónico debido a que se trata a menudo de testimonios individualizados. Lamentablemente, hasta el momento, escasea la información sobre la visión y la experiencia africana de la esclavitud. No cabe duda que un examen contínuo y cada vez más detallado de la cuestión esclavista desde una perspectiva local, como ha sucedido el caso norteamericano ${ }^{20}$, bien sea a través de documentos europeos, podrá ir arrojando indicios sobre el lado más afectado por el sistema esclavista.

\section{Breves apuntes sobre el esclavismo tardío español}

Por esclavismo tardío español tendríamos que entender el que se llevó a cabo en las últimas colonias españolas a partir de la segunda década del siglo XIX una vez emancipados los territorios continentales y que reúne unas características propias diferentes al esclavismo previo. El esfuerzo de recopilar y estudiar las historias locales en España en su relación con el sistema esclavista, debe ayudar paulatinamente a componer un retrato mucho más riguroso que el existente actualmente sobre la importancia real o relativa de esta economía, su sistema jurídico y de ideas tanto para el conjunto de España como para la propia América y África.

En lo referente a la economía esclavista, en primer lugar es conveniente trazar la cadena de actores implicados que principia con el grupo de actores encargados de la captura de personas. Estos solían ser desde los inicios en el siglo $\mathrm{XV}$ jefes y reyes africanos alentados por las recomendaciones y acciones de tratantes europeos asentados en suelo africano, a vender los cautivos de sus guerras y razias con reinos vecinos. Este sistema viciado de principio iría degenerando a partir del siglo XVIII en una carrera interafricana de autoridad y conquista, apoyada en el uso de armas de fuego europeas introducidas por los tratantes y fuertemente influida por los intereses de estos europeos, a la que se sumarían en ocasiones personas esclavizadas por deudas o por la arbitrariedad hacia sus propias poblaciones de algunos jerarcas africanos, generalmente influidos por agentes europeos.

El ámbito de acción preferente de los tratantes europeos, denominados Mongos, fue desde el siglo XV la costa de la denominada Guinea una larga franja de

20. El libro de Kenneth Stamp, La esclavitud en los EEUU, Oikos-Tau edición española: Vilassar de Mar Barcelona 1966. Deconstruye mediante el estudio de documentos de hacendados estadounidenses ya en los años 60 del siglo XX el discurso proesclavista de los estudios precedentes al ofrecer numerosos testimonios sobre visión de los propios esclavizados que se desprende de dicha documentación. 
tierra que va del Senegal al Congo en el África Occidental, aunque a partir del primer tercio del siglo XVIII, adquiere también importancia el área de Mozambique y la isla de Madagascar en las costas orientales. Las rivalidades de las potencias europeas condicionaban el lugar y el modo en que se obtenían los prisioneros y en el contexto de estas rivalidades. España que se había mantenido en cierta medida al margen de las operaciones en África, inicia con grandes dificultades derivadas sobre todo del contexto de Guerra europeo a principios del XIX, su penetración en el continente, con el impulso de la Real Cédula de 28 de febrero de 1789 que liberalizaba la introducción de personas esclavizadas procedentes de África, sustituyendo el anterior sistema de asientos o concesiones. Posteriormente diversas Reales Órdenes se fueron superponiendo hasta 1821, con el objeto de facilitar la introducción de mano de obra esclavizada en las colonias.

Con el tiempo se irían perfilando como lugares de trata preferente de los buques españoles las zonas de La costa de la Pimienta, concretamente el río GaIlinas, donde operó desde 1822 el malagueño Pedro Blanco uno de los Mongos más destacados del siglo XIX ${ }^{21}$, las zonas de Widah, Lagos y finalmente el área de Corisco en la actual Guinea Ecuatorial.

El segundo actor del sistema esclavista es el comerciante implicado en la contrata con las autoridades africanas o con los mongos, e implicado asimismo en el transporte de las personas esclavizadas a las colonias. Los comerciantes esclavistas conocidos como negreros, buscaban el enriquecimiento rápido con métodos nada piadosos. Si cruel e injusto era el sistema esclavista en su totalidad más crueles eran los procedimientos de transporte de las personas. Se llegó a afirmar que en un buque negrero se concentraban todos los crímenes de la humanidad $^{22}$, pues estaban presentes el rapto, el asesinato, la violación, la tortura, la privación de libertad. No era extraño arrojar personas sanas por la borda en previsión de contagios o por haber subestimado las necesidades alimenticias ante una calma en alta mar y lo que es aún más despiadado, en ciertos casos documentados se arrojaban a todas las personas por la borda ante la inminencia de una captura por parte de los buques ingleses que a partir de la prohibición de la trata, se encargaban de la represión del tráfico de personas o simplemente para cobrar el seguro sobre el cargamento del buque ante una singladura que se presentara difícil23.

En el transporte de personas esclavizadas debemos tener en cuenta los siguientes individuos: por una parte el comerciante naviero que podía ser uno o

21. José Luciano Franco, Comercio Clandestino de Esclavos, Editorial de Ciencias Sociales, La Habana, 1980.

22. M. Barón Fortacín, Cuestión de Cuba: La abolición de la esclavitud, Madrid 1879. En: facsimil de la primera edicion por; Universidad Politécnica de Madrid 1993 pp. 15-16 citando a John Wesley y Lord Palmeston.

23. M. Barón Fortacín, Cuestión de Cuba: La abolición de la esclavitud, Madrid 1879. 
estar asociado en régimen de participación y ganancia proporcional o bien tratarse de una empresa con accionistas, luego la tripulación del barco, capitán, piloto y marinería y finalmente los agentes de los puertos de recepción en las colonias que a menudo poseían barracones de internamiento para la redistribución de las personas esclavizadas a diferentes puntos. El último tramo de la cadena esclavista lo constituyen los hacendados que realizaban la compra de las personas y que mediante la presión de capataces exprimían al máximo su trabajo. Sin embargo no debemos dejar de tener en cuenta la existencia de terceros beneficiados más o menos directamente del tráfico de personas esclavizadas y del sistema esclavista en su conjunto. Entre estos terceros estarían las compañías aseguradoras de la carga de los buques, y todos los implicados en la producción de los efectos demandados en África por los vendedores de personas esclavizadas. Estos productos que abarcaban una enorme variedad, podían ser desde trozos de hierro y alambre a piezas de tela, ron, armas de fuego, pero sobre todo la moneda africana más reputada el cauri (Cyprae moneta), un caracol marino procedente del índico y cuya extracción pronto controlaron los ingleses. Es de apreciar que estos productos en especial el cauri tenían un coste marcadamente inferior a la ganancia que por ellos se obtenía que era superior a 25 sobre $1^{24}$, siendo esta la principal razón de la rentabilidad de las operaciones de la trata, a pesar de producirse con el tiempo una enorme inflación compensada empero por el aumento de los precios de venta de las personas esclavizadas en época de represión de la trata.

El sistema esclavista español empezó a sufrir importantes cambios a raíz de acontecimientos internacionales que condicionaron la salida de Inglaterra de dicho sistema. El primero de estos acontecimientos, puede señalarse, fue la emancipación de las colonias inglesas de norte América, con la emancipación de las colonias inglesas más importantes en el continente Americano, empezó a perder interés el comercio de esclavizados para los ingleses, comercio que se vio cada vez más denostado por la opinión pública interna inglesa que no encontraba motivos para continuar con lo que se reputaba como un crimen contra la humanidad y una vergüenza para las naciones que se gloriaban de "civilizadas". Por el Acta de Plymouth de 25 de marzo de 1807 quedaba prohibida toda importación de personas esclavizadas sobre territorios dependientes de la Corona británica. Seguidamente se observa un enorme descenso en la importación de personas esclavizadas en las colonias españolas. En Cuba se pasó de introducir 13.832 personas esclavizadas en 1802, en un momento de auge tras la paz de Amiens, a 1.162 en 1809, tal importancia revestía la nación inglesa en el comercio. Este descenso lejos de desalentar a los implicados en el sistema esclavista español los alicientó aún más en una búsqueda de víctimas que esclavizar, esta vez sin intermediarios que redujesen los beneficios.

24. Juan Perez de La Riva, "Nota sobre las monedas usadas en la costa de África durante el siglo XVIII" en Revista de la Bibliotaca Nacional José Martí. 
En este contexto irrumpe con una fuerza extraordinaria la represión inglesa del tráfico de personas esclavizadas, ya desde el año 1809, en que de 24 barcos esclavistas que partieron de la Habana rumbo a África 9 fueron apresados por los ingleses ${ }^{25}$. Esta cuestión de la represión inglesa arrastra desde sus inicios hasta hoy encendidos debates, por una parte están quienes defienden que con la represión ejercida sobre otras naciones, Inglaterra pretendía obtener el beneficio de estorbar el comercio de esas naciones, mientras que otros autores ponen el acento en una verdadera causa inglesa en pro de la abolición universal de la trata y más adelante de la propia esclavitud. Sobre este particular que excede los propósitos del presente estudio, cabe apuntar que si bien Inglaterra obtuvo beneficios por la persecución del tráfico de esclavos como el afianzamiento de su posición en África, o la conquista de una posición de privilegio en el comercio internacional, no es menos cierto que esos beneficios se derivan del hecho mismo del cambio de producción esclavista al modo de producción capitalista, de lo que se infiere que cualquier nación que hubiese tomado la prevención en su día de abolir el tráfico de esclavos y la esclavitud antes que otras, como de hecho hicieron los daneses, hubiera obtenido similares beneficios que los obtenidos por Gran Bretaña. Resulta cuanto menos triste ver como algunos autores españoles hoy todavía, se rasgan las vestiduras ante lo que llaman el acoso inglés, haciéndose eco de la mentalidad más ramplona del siglo XIX. Lo cierto es que aunque España se sumó a los tratados de prohibición del tráfico de esclavos en 1817 parcialmente y luego en 1820 con disposiciones que lo hacían efectivo a partir de octubre de ese año, nada hizo por procurar que se cumpliese, bien al contrario permitió con total indulgencia que creciese el tráfico antillano avivado por el incremento de precio sobre las ventas que la misma represión inglesa producía.

La dicotomía a la que se enfrentaban las autoridades españolas era la de disponer en contra de la metrópoli a los hacendados de las últimas colonias que le quedaban a España, frente a cumplir con el deber de limpiar el nombre del país ante los ojos de las naciones de Europa y los sectores liberales del interior. En 1826 el gobierno español dicta unas disposiciones para la persecución de la trata, presionados por el gobierno británico que amenazó con sublevar la isla de Cuba, pero estas fueron letra muerta, pues con la complicidad de las autoridades cubanas y en especial el Capitán General de la Isla el general Francisco Dionisio Vives, el tráfico continuó y tuvo momentos de auge ${ }^{26}$. El comercio clandestino adquiría tales proporciones que incluso el Administrador de Rentas Reales de Trinidad llegó a proponer al Intendente de Hacienda de Puerto Príncipe que se obtuviesen beneficios en favor de la Real Hacienda por este comercio ${ }^{27}$. Para hacerse una idea de la magnitud del tráfico ilegal tengamos en cuenta que si en 1774 exis-

25. José Luciano Franco, Comercio Clandestino de Esclavos.

26. José Luciano Franco, Comercio Clandestino de Esclavos.

27. Véase en José Luciano Franco pp. 327-328. 
tían en Cuba 44.330 personas en esclavitud, en 1841 fueron 436.459 y esta cifra sólo empieza a descender en la segunda mitad del siglo XIX existiendo en 1862, 368.550 personas esclavizadas. A estas cifras debemos añadir que el crecimiento de la población esclavizada era negativo por la alta tasa de masculinidad y por la espantosa mortandad que en algunas haciendas fue del 10\% anual.

Gran Bretaña desde la abolición del tráfico de personas esclavizadas se lanzó a una campaña internacional en Europa, África y América de la que se obtuvieron tratados conjuntos y compromisos tanto de potencias occidentales como de reinos africanos, se nombraron comisiones mixtas para juzgar a los barcos apresados, entre las que la española tenía sus sedes en Cuba y en Sierra Leona. Asimismo se comprometió Inglaterra a indemnizar a España por 10 millones de pesetas. Mientras, en Cuba, la situación no cambiaba y los sucesivos capitanes generales se entregaban a tratos con los traficantes en complicidad con los hacendados. Por otra parte los navieros implicados en la trata mejoraban sus técnicas de disimulo, como se observa por ejemplo en las instrucciones dadas al capitán de la "Semirámide" por Roig Sobrino y Compañía, donde se indican los lugares apropiados de desembarco en Cuba y donde se puede entrever la connivencias de las autoridades cubanas ${ }^{28}$.

Las autoridades Británicas obtuvieron de la España de la regencia de $M^{\mathrm{a}}$ Cristina el tratado de 1835 que inauguraba una etapa en la que existirían instrumentos legales para la supresión de la trata, una modificación adicional hacía que el país que apresara un buque traficante de personas esclavizadas sería el responsable de la emancipación de los esclavizados. Esto hacía que en la práctica el gobierno inglés fuera el encargado de emancipar a todos los esclavizados procedentes de capturas evitándole a España la embarazosa situación de introducir libertos en una colonia como la cubana cuyas elites eran tan afectas al sistema esclavista. Aún así la comisión de la Habana sólo condenó a 4 barcos entre 1835 y 1870 mientras que en Sierra Leona únicamente entre 1845 y 1862 se condenaron 189 y 3 fueron absueltos ${ }^{29}$.

El 9 de enero de 1845 se redactó una nueva ley que penaba a todos los implicados en el tráfico; propietarios, armadores y dueños del cargamento, aunque de hecho rebajaba las penas del capitán, compradores, maestres y pilotos respecto a la real orden de 1817 . Todavía en 1845 se escuchan defensas en el Congreso de Diputados como la del Sr. Olivan Diputado en las que se dice que la esclavitud debe "protegerse mejorarse y consolidarse" (sic) a pesar de que unas frases atrás se dice "la compadezco y la repruebo" 30 . Todo ello es sintomático de la esquizofrenia política ante el temor del desastre que supondría perder los últimos baluartes del moribundo imperio español.

28. Mariano L. Castro y $M^{\underline{a}}$ Luisa de la Calle, Origen de la colonización española de Fernando Poo, Secretariado de publicaciones de la Universidad de Valladolid, Valladolid 1992, pp. 117-119.

29. Mariano L. Castro y $M^{a}$ Luisa de la Calle, Origen de la colonización española de Fernando Poo.

30. Íbid 26. 
A pesar de las disposiciones siguientes, en especial la de 1867, todavía continúo el tráfico. Hasta fecha tan tardía como el 25 de enero de 1870, en que eran desembarcadas en Cuba 600 personas esclavizadas procedentes de África y aún más tarde un barco con niños esclavizados ${ }^{31}$.

Distintos fueron los derroteros que siguió la abolición de la esclavitud propiamente, si bien en la España peninsular diferentes Reales Cédulas iban a lo largo de los siglos XVII y XVIII concediendo emancipaciones a esclavos fugitivos de otros Estados, no es hasta Real Orden de 18 de agosto de 1859 que se afirma que el título de propiedad sobre un esclavo no tiene efecto en la península. Orden que se hizo extensiva en 1865 al esclavo fugado procedente de Cuba que viniese a la España peninsular. En fecha tan escandalosamente tardía como 1868 se declara que los nacidos de mujer esclava a partir del 17 de septiembre eran libres. Deben remarcarse en este contexto como causas, por una parte los temores a que una insurrección general de los esclavizados en Cuba acabase por dar al traste con todo el sistema esclavista, como venía sucediendo en Haití, pero también en las propias Cuba y Puerto Rico y por otro lado los esfuerzos de sociedades abolicionistas y católicas españolas.

No fue hasta 1886, con la derogación de las últimas disposiciones sobre trabajo forzado que se pueda afirmar que todos los esclavizados en territorios bajo dominio español fueron formalmente libres.

\section{Repercusión en Mallorca de la cuestión abolicionista}

Un estudio que es de vital importancia que se emprenda para conocer, la realidad social y económica de la isla es la continuidad de la esclavitud más allá del siglo XVII en que está atestiguada. Se ha establecido la existencia de una esclavitud importante de personas negras en España en el siglo XVI, un estudio clave en este sentido es el de José Luis Cortés López ${ }^{32}$, pero apenas se ha avanzado sobre la cuestión más allá del XVII. Existió asimismo una importante esclavitud originada en las razias del corso sobre el norte de África. Todavía en el siglo XVIII hallamos en Mallorca cautivos de corso, 22 en 1710 y 25 en $1711^{33}$ :

Ahora bien no podemos estar seguros de la evolución posterior de la situación, aunque, es común afirmar que en la España peninsular la esclavitud desapareció de hecho en 1766, al comprar un enviado del sultán de Marruecos la libertad de los esclavizados musulmanes de Sevilla, Cádiz y Barcelona. Nuestra hipótesis inicial sería que la esclavitud derivó a formas serviles como la existencia de criados altamente dependientes de sus señores, con derechos teóricos pero no en la praxis.

31. Artuto Barrera Arnalte, Los últimos esclavos de Cuba. Los niños cautivos de la goleta Batans, Alianza Ed. 2001.

32. José Luis Cortes López, La esclavitud negra de la España peninsular del siglo XVI Universidad de Salamanca 1989.

33. Andreu Bibiloni Nadal, el comerç exterior a Mallorca. Homes, mercats i productes d'intercanvi 1650-1720. El tall del Temps, Mallorca 1995. 
No hemos hallado presencia de esclavitud en el XIX en Mallorca, pero hay que hacer constar la importante cantidad de criados que se reseñan en el censo de Floridablanca ${ }^{34}$, este bien podría haber sido el subterfugio con el que camuflar una servidumbre esclavista o pseudo-esclavista, sobre todo si tenemos en cuenta las disposiciones citadas más arriba sobre "esclavos venidos a la península". Asimismo hallamos algunos apuntes que deben ser investigados sobre licencias de embarque relacionadas con Mallorca ${ }^{35}$. Debemos considerar que hasta épocas bien recientes grupos de personas marginadas vivían en total dependencia de señores y con poco contacto social con el grupo mayoritario, esto incluiría con bastante probabilidad toda minoría étnica, como lo demuestra, por ejemplo, la pervivencia de fenotipos negros en Huelva.

En este estado de cosas y esperando la ocasión de poder avanzar al respecto, quedan otras cuestiones que reseñar, como la repercusión de las leyes abolicionistas o de represión de la trata en la sociedad de estudio, la mallorquina. Para avanzar en este propósito hemos recogido dos documentos que juzgamos de importancia. El primero de estos documentos es el expediente de 1842 de la junta de Comercio de Mallorca ${ }^{36}$, promovido con relación al expediente presentado por la Junta de Comercio de Cataluña a instancias del tribunal de Comercio de la Habana y a través del Tribunal de Comercio de Cataluña, en que se eleva una protesta ante la propuesta del gobierno inglés de control directo, mediante un Superintendente, de la emancipación de esclavos y para la emancipación de todos los venidos desde el año 1820, procedentes de capturas de barcos esclavistas en las colonias españolas. Del expediente catalán, ofrecemos los siguientes extractos: el tribunal de Comercio sobre la protesta cubana:

...El documento á que nos referimos toma por base de sus raciocinios el temor de que nuestro Congreso se empeñe inconsideradamente en la cuestión de la esclavitud; y en realidad si esto aconteciese, la sola discusión sería una señal de terrible alarma para nuestras posesiones ultramarinas, porque aquellos habitantes, recelosos de la inesperiencia y ardimiento declamatorio que acompañan generalmente semejantes debates, verían en ellos el origen de una lucha estemporánea; pero que plasmaría el espíritu de empresa y la confianza se estinguiría en aquellos países, hasta el punto de provocar la emigración de los blancos con sus capitales.

34. Josep Juan Vidal, El cens de Floridablanca a les Illes Balears, 1786-1787, Miquel Font, Palma de Mallorca 1989.

35. Véase el oficio del juez de Arribadas de Mallorca que remite solicitud y minuta de real orden que aprueba las licencias a siete pasajeros que partieron junto con Bartolomé Moragues, en el bergantín "Nuestra Señora del Carmen" alias "el Dión", en Archivo General de Indias INDIFERENTE, 2155, N. 139.

36. Archivo del Reino de Mallorca: Junta de Comercio, Expedientes, Caja 44 no 451. 
Y más adelante:

...Este es el pretexto (el control de la emancipación de esclavizados) con el que la poderosa Albión quiere infiltrarse, por decirlo así, en todas las cuestiones futuras que puedan ocurrir sobre la libertad de los esclavos.

En cuanto al dictamen de la Junta de Comercio de Cataluña:

...por último la junta debe pedir enérgicamente al Gobierno Superior, que evacuados los informes pendientes en la isla de Cuba sobre este negociado ponga un término a semejantes exigencias (las inglesas) aunque sea declarando piratería el Comercio Africano" y antes: "...Este es uno de aquellos incidentes, que es muy difícil tratarlo con templanza: sería preciso no tener una gota de sangre española en las venas para no sentir cierto corage é indignación al ver hasta que punto se quiere envilecer y ultrajar la independencia nacional.

De la resolución primera de la Comisión de Competencia de la Junta de Comercio de Mallorca se transcribe a continuación:

La comisión de Competencia en cumplimiento del acuerdo de V.S de 7 de los corrientes se ha ocupado seriamente del contenido de las representaciones é informes que la Junta de Comercio de Cataluña ha dirigido al Gobierno acerca el proyecto que ha propuesto el de Inglaterra para la emancipación de esclavos de las colonias españolas. Y convencida la misma Comisión de que son fundadas y poderosas las razones que en contra del proyecto presentado se exponen en los documentos que van expresados, es de dictamen: que V.S. en bien general de la nación y particular de estas islas y accediendo a los deseos de la Junta de Cataluña debe unir sus votos a los de aquella para que sean atendidas sus justas reclamaciones en el particular que se trata: y que podrá V.S si gusta, verificará por medio de la representación de S.A.S. el Regente del Reino, cuya minuta sigue a continuación...

Esta cuestión tan encendida tendrá su eco en la prensa Mallorquina con artículos en el Diario Constitucional de Palma de 2 de enero, 7 de enero, 9 de enero y 13 de enero de 1842. En estos artículos se inflaman en una espiral los sentimientos de "nación ultrajada" y se hace gala de una importante dosis de cinismo, al afirmar que se quiere abolir la esclavitud mientras que por otra parte nada se hace en ese sentido si no es por presión inglesa. El segundo documento al que nos referimos es el "Expediente de 1844 sobre las instrucciones dadas a los oficiales de la marina inglesa encargados de la persecución del tráfico de negros" que Resuelve la Junta de Comercio de Mallorca favorablemente, y manda se publiquen las resoluciones del Senado y la Real Orden subsecuente en el Dia- 
rio Constitucional de Palma y en el Genio de la Libertad también de Palma, ambos el 19 de enero de $1845^{37}$.

De estos expedientes se desprende que los comerciantes de Mallorca basculan entre las pasiones comunes del momento en España. El ardor fanático que se inflama pronto, tiene su base en un temor no ocultado a la independencia de Cuba. La experiencia de la independencia de la América continental sirve de ejemplo (en los ejércitos de Bolívar no menos de la mitad de las tropas eran personas negras que buscaban la libertad). En esa línea merece especial atención la expresión "en bien general de la nación y particular de estas islas" de la resolución de la Comisión de Competencia de la junta de comercio que, caso de referirse a las Baleares como parece y no a Cuba y Puerto Rico, explicita que no es sólo una cuestión de opinión la que aquí se dirime sino de interés propio y común (de los libres claro está). Interés que en este caso, no se nos oculta, pasa por el mantenimiento de los lazos coloniales y por ende comerciales, y más por el mantenimiento de los intereses indisociables de los primeros e involucrados en la trata y en la economía de explotación esclavista propiamente.

\section{Noticia sobre un barco esclavista mallorquín}

La escasez de referencias a la participación en el tráfico de esclavos por parte de mallorquines y Baleáricos en general, indica más una intencionalidad por parte de autores e investigadores en obviar u ocultar información referente a la cuestión que un pretendido desinterés. Recientemente la prensa balear se ha hecho eco de la investigación que establecía el origen ibicenco del piloto de la famosa goleta "La amistad" 38 El motín de esclavizados de "La amistad" ha sido Ilevado al cine por Steven Spielberg ${ }^{39}$. Este inusual interés del cine y de la prensa por un capítulo concreto de la historia decimonónica indica que el tema no deja de ser sensible. Si en el caso catalán tenemos ampliamente establecida su presencia en la trata gracias, entre otros trabajos, a los preciados artículos del número 75 de $L^{\prime} A v e n c ̧{ }^{40}$, y aunque siguen faltando estudios en profundidad, podemos decir que existen paralelismos y conexiones comerciales ente Cataluña y Baleares que permiten afirmar que existieron puntos de conexión en el criminal comercio.

37. Archivo del Reino de Mallorca: Junta de Comercio, Expedientes, Caja 44 no 451 .

38. Michael Zeuske Orlando García Martínez, La Amistad de Cuba, Ramón Ferrer, contrabando de esclavos, captividad y modernidad atlántica. Caribbean Studies, Vol. 37, Núm. 1, enero-junio, 2009, pp. 119-187 Universidad de Puerto Rico, Puerto Rico citado en el Diario de Ibiza, "Joan Planells, Antoni Ferrer Abárzuza y Pere Vilàs siguen los pasos del marino..." Jueves 26 de enero de 2012.

39. Steven Spielberg, Amistad (película) 1997.

40. El número 75 de L'Avenç (octubre de 1984) presenta seis dossieres de: Josep M. Fradera, Màrius Armengou, J. Rovira i Fors, Josep M. Fradera, Manuel Moreno Fraginals, y Rebbeca J. Scott sobre la economía esclavista en el Caribe y su relación con Cataluña. 


\section{Afirma Josep Fradera:}

no existió empresa catalana de dimensiones medianas que no haya organizado o participado en la financiación de empresas negreras ${ }^{41}$.

Para este autor entre las familias y personajes dedicados al infame negocio destacan los Roig, Vidal, Samá, Miró i Pic, Ignasi Carbonell, Panxo Martí (un mongo), Pau Forcadé, J. M ${ }^{a}$ Borrell, Miquel Pous, la casa Prats, Pujol i Cía. El armador catalán Josep Vidal i Ribas tenía incluso habilitada una factoría en Widah en el golfo de Biafra. En busca de estos nombres he tenido la fortuna de hallar las valiosas informaciones contenidas en la página electrónica dedicada al Sargento Naval William Loney ${ }^{42}$ entre cuyo currículo consta el haber pertenecido al escuadrón encargado de reprimir el tráfico de esclavizados en África Occidental. En el informe de dicho escuadrón del año 1845 se dice de los barcos capturados:

Dos de los barcos españoles tenían pasaporte (matrícula) de Matanzas, tres de Santiago de Cuba, la Habana y Palma en Mallorca; dos tenían viejos pasaportes (matrículas) portugueses datados en Lisboa y dos no tenían papeles oficiales.

Del número total veintidós fueron capturados al Norte y ocho al Sur del Ecuador; diecisiete eran de construcción americana $\left({ }^{43}\right)$, ocho de construcción brasileña y cinco de construcción española ${ }^{44}$.

En el Anexo 2 del presente artículo, se ofrece la lista de barcos y en el Anexo 3 se ofrece el informe al completo. Teniendo como referencia las publicaciones de Joan Pou Muntaner ${ }^{45}$ y de Ramón Sampol ${ }^{46}$ intentamos trazar una posible coincidencia de nombres de barcos. En efecto, de los nombres mencionados en ambas obras, que incluyen si no todos, sí una parte importante de los veleros de Baleares del siglo XIX y en especial los dedicados al comercio de largas distancias, hallamos una coincidencia posible en fecha. Se trataría del San Antonio;

En 1838 emprenen la ruta de les Antilles... els quetxos Tres Hermanos i San Antonio... ${ }^{47}$

41. Josep M. Fradera, "Catalunya i Cuba en el segle XIX: El comerç d'esclaus", L'Avenç no 75 octubre 1984 .

42. http://home.wxs.nl/ p pdavis/ dirgida por Peter Davis Zeist.

43. En Estados Unidos, se deduce.

44. Texto original: "Two of the Spanish vessels had their passports dated at Matanzas, three at St. Jago de Cuba, Havana, and Palma in Majorca; two had old Portuguese passports, dated in Lisbon, and two were without official papers. Of the whole number twenty-two were captured to the north, and eight to the south of the Equator; seventeen were American-built, eight Brazilian, and five Spanish.".

45. Joan Pou i Muntaner, El comerç amb les Amériques, Gráfiques Miramar, Palma de MaIlorca 1991.

46. Ramon Sampol Isern, Velers de les Balears, Ed. Miquel Font, Palma de Mallorca 1986.

47. Joan Pou i Muntaner, El comerç amb les Amériques, Gráfiques Miramar, Palma de MaIlorca 1991 p. 12. 
Sin embargo y a pesar de las coincidencias de Fecha aproximada, nombre del barco, ruta y procedencia, debemos avisar que "San Antonio" es común denominación para embarcaciones del momento. Como muestra sirva decir que en la lista de buques esclavistas proporcionada por Curtin, Philip D. and Herbert S. Klein $^{48}$ se menciona no menos de seis buques esclavistas portugueses con el nombre Santo Antonio para el período de 1817 a 1843, es cierto que ninguno Español. No podemos pues concluir que sea el San Antonio el barco referido, máxime si consideramos la inconsistencia en el tipo de barco, puesto que un queche es un barco de un sólo palo y en ningún caso puede ser confundido con un bergantín. Queda de todos modos establecido que en el informe de 1845 del escuadrón inglés encargado de reprimir el tráfico esclavista en las costas africanas se afirma haber detenido un buque con documentación del puerto de Palma de Mallorca. Es seguro que avanzarán los investigadores interesados en la cuestión, si se cotejan los datos existentes sobre entradas y salidas de buques MaIlorquines en los puertos coloniales. Ante el panorama de al menos un barco apresado no será de extrañar que se pueda desvelar fácilmente la existencia de otros varios entregados al nefando negocio.

\section{Conclusión}

Los elementos para el estudio de la relación de Mallorca en particular y de las Islas Baleares en general con el esclavismo atlántico son todavía dispersos. Por una parte la presencia de gran cantidad de datos sobre esclavitud en archivos históricos de Puerto Rico así como la redacción discontínua de estudios monográficos localistas y biografías de familias de hacendados deberán ponerse en relación para establecer un retrato general y riguroso del panorama general. El criminal sistema esclavista implica diferentes actores en distintas etapas y fases que deben ser diferenciadas. Para valorar la influencia en Mallorca de dicha economía se debe estudiar tanto la influencia de Mallorquines entre los propietarios de haciendas esclavistas como su presencia entre los comerciantes de personas esclavizadas, sin descuidar la atención sobre los emprendedores e inversores relacionados con la esclavitud. Para lo anterior es cruciar atender a la desfavorable opinión local, visible en la prensa de Mallorca, ante cuestiones que afectan a la economía esclavista decimonónica como el abolicionismo o la represión de la trata. También el cotejo de los datos existentes sobre barcos esclavistas como el caso de la Goleta "La Amistad" (en ese caso ibicenca) y los barcos apresados por las autoridades británicas en su función de represión de la Trata, promete aportar información relevante al objeto de esta cuestión. De lo estudiado

48. Curtin, Philip D. and Herbert S. Klein, Records of Slave Ship Movement Between Africa and the Americas, 1817-1843 [machine-readable data file]. 2nd DPLS ed., 1973, 1997. Madison, WI: Data and Program Library Service [distributor]; http://dpls.dacc.wisc.edu/slavedata/ slaintro1.html. (14 June 1999). 
hasta el presente se desprende una participación activa y destacada de hacendados provenientes de la población de Sóller principalmente en Puerto Rico y secundariamente en Cuba, así como indicios de la importancia de la presencia de hacendados mallorquines en Venezuela. La imagen negativa de los mallorquines también es clave en el levantamiento de los esclavizados y de un modo olvidado ha sido nuclear en las raíces del movimiento independentista de Puerto Rico. La preocupación por la presencia mallorquina en el esclavismo atlántico debe servir para coadyuvar en el esfuerzo de establecer, con una precisión mayor que la actual, las relaciones de regiones concretas de España en el sistema esclavista. El conocimiento preciso de historias específicas, particularmente a través de las biografías de los esclavistas mallorquines contribuirá a aclarar un lado de la historia, la economía y el drama esclavista. Pero no debe perderse de vista que ahondar en las historias locales del esclavismo español debe necesariamente ofrecer información sobre la visión más desconocida, la de los propios africanos y africano-descendientes, que para el mundo hispánico todavía apenas puede inferirse de un modo indirecto. 
ANEXO 1. Censos y documentos relativos a la esclavitud en: http://www.preb.com.

\begin{tabular}{|l|l|l|}
\hline Municipalidad & Año & Documento \\
\hline Barros (Orocovis) & 1833 & Esclava compra su libertad \\
\hline Bayamón & 1824 & Fuga de esclavos de la hacienda de Juan Sánchez \\
\hline Coamo & 1872 & Dueños de esclavos, num. de esclavos, barrios \\
\hline Corozal & 1870 & Gráfica: oficios de los 85 esclavos clasificados por barrios \\
\hline Corozal & 1870 & Gráfica: niños y adultos esclavos clasif. por su estado civil \\
\hline Corozal & 1870 & $\underline{\text { Lista de dueños de esclavos }}$ \\
\hline Corozal & 1871 & Gráfica: esclavos clasificados por oficios \\
\hline Hormigueros & 1867,1871 & $\underline{\text { Dueños de esclavos, haciendas, barrios }}$ \\
\hline Moca & 1826 & $\underline{\text { Propietarios de esclavos }}$ \\
\hline San Germán & 1867 & $\underline{\text { Hacendados por barrios, esclavos }}$ \\
\hline San Germán & 1871 & Dueños de esclavos, esclavos clasificados por sexo \\
\hline Varios & 1872 & Dueños de escl. de varios pueblos felicitados por el Rey \\
\hline Vega Baja & 1838 & $\underline{\text { Lista de hacendados, núm. de esclavos, cuerdas }}$ \\
\hline
\end{tabular}

Fuente: Elaboración propia a partir de los datos de "PReb" (Puerto Rico en breve) http://www.preb.com.

ANEXO 2. La lista muestra los 9 barcos capturados y condenados por tráfico de esclavos por la comisión mixta británico española de Sierra Leona en 1845.

\begin{tabular}{|c|c|c|c|c|c|c|}
\hline $\begin{array}{c}\text { Fecha de } \\
\text { captura } \\
(1845)\end{array}$ & $\begin{array}{l}\text { Nombre del } \\
\text { buque }\end{array}$ & $\begin{array}{c}\text { Tipo de } \\
\text { embarcación }\end{array}$ & Constructor & $\begin{array}{l}\text { Número de } \\
\text { esclavizados }\end{array}$ & \multicolumn{2}{|c|}{ Lugar de captura } \\
\hline 11 Ene. & \begin{tabular}{|c|} 
San Antonio, \\
alias El Cayman
\end{tabular} & Bergantín & Growler & Sin datos & $714 \mathrm{~N}$ & $1236 \mathrm{~W}$ \\
\hline 3 Feb. & Sua Majestade & Goleta & Albert & 421 & $1010 \mathrm{~N}$ & $1430 \mathrm{~W}$ \\
\hline 11 Feb. & Triumfo & Bergantín & Albert & Sin datos & $1018 \mathrm{~N}$ & $155 \mathrm{~W}$ \\
\hline 13 Feb. & Venus & Goleta & Albert & Sin datos & \multicolumn{2}{|c|}{ Rio Pongas } \\
\hline 24 Feb. & Huracan & Falucho & Hydra & 70 & $52830 \mathrm{~N}$ & $15720 \mathrm{E}$ \\
\hline 4 Mar. & Pepito & Falucho & Hydra & 312 & $6730 \mathrm{~N}$ & $14550 \mathrm{E}$ \\
\hline 25 Mar. & Dos Hermanos & Bergantín & Ardent & Sin datos & \multicolumn{2}{|c|}{ Rio Pongas } \\
\hline 15 May. & Mariana & Bergantín & Éclair & Sin datos & \multicolumn{2}{|c|}{$\begin{array}{l}\text { Fuera de Gargbah, } \\
\text { cerca del Gallinas }\end{array}$} \\
\hline 23 May. & Venganza & Schooner & Waterwitch & Sin datos & $545 \mathrm{~N}$ & $105 \mathrm{~W}$ \\
\hline
\end{tabular}

Fuente: http://home.wxs.nl/ pdavis/ (Página Web dirigida por Peter Davis Zeist). 
ANEXO 3. Informe ingles de 1845 sobre el estado de la Represion del Tráfico Esclavista en la Costa Occidental Africana. M.L. Melville y J. Hook "Commisioners Report".

Her Majesty's Commissioners to the Earl of Aberdeen.

Sierra Leone, December 31, 1845. (Received February 24, 1846.)

My Lord,

We have the honour to enclose herewith a list of all the cases adjudicated during the year 1845, in the British and Brazilian Court of Mixed Commission, and in the British and Spanish Mixed Court of Justice, established in this colony.

No case came before the British and Netherlands, British and Argentine, British and Chilian, British and Bolivian, nor the British and Uruguayan Mixed Courts of Justice.

The number of cases adjudicated was thirty, of which nine were tried in the British and Spanish Court, and twenty-one in the British and Brazilian Court. Twenty-eight were cases of condemnation, and two were dismissed, having occurred subsequently to the 13th of March last, at which period the Convention of 1817 , under which they were captured, terminated.

1189 slaves were emancipated during the year, of whom 1187 were registered. (During the same period 2,032 slaves, the survivors of 2,829 captured, were emancipated by the Court of Vice-Admiralty at Sierra Leone. These slaves were found on board of four vessels, all engaged in the Brazilian trade, but one of them only furnished with papers.)

The total number of vessels prosecuted before the Mixed Commissions, since their establishment in this colony in June 1819, up to the present date, is 528, whereof 501 were cases of condemnation, and twenty-seven were either withdrawn, dismissed, or restored to the claimant.

During the same period there have been emancipated by these Courts, 64,625 slaves, of whom 56,935 have been registered here.

Of the vessels adjudicated during 1845, three Spanish and one Brazilian had slaves on board when captured.

Two of the Spaniards had shipped their cargoes at Popoe for conveyance to Brazil, the other at the Pongas, and was bound to Havana.

The Brazilian vessel embarked her slaves at Lagos for delivery in the neighbourhood of Rio de Janeiro.

The destinations on the coast of the other twenty-six vessels, were three for Rio Pongas, three for Gallinas and New Cestos; twelve for Lagos, Whydah, and Popoe, in the Bight of Benin; and eight for Loango, River Congo, Cabinda, Ambriz, and Angola: their return voyages, so far as can be ascertained, were to 
have been, five for the Island of Cuba, ten for Bahia, seven for Rio de Janeiro and Cape Frio, and four for Pernambuco, Santos, and Campos.

Of the twenty-one vessels adjudicated in the British and Brazilian Court, five had no passports, four had them dated at Rio de Janeiro, nine at Bahia, two at Pernambuco, and one at Santos.

Two of the Spanish vessels had their passports dated at Matanzas, three at St. Jago de Cuba, Havana, and Palma in Majorca; two had old Portuguese passports, dated in Lisbon, and two were without official papers.

Of the whole number twenty-two were captured to the north, and eight to the south of the Equator; seventeen were American-built, eight Brazilian, and five Spanish.

Nearly all the remarks made in the report of last year, sent from these Mixed Commissions, apply with equal force to the state of the Slave Trade on this coast during 1845.

In the immediate vicinity of this colony - in the Rio Pongas to the northward, the Sherbro and Cape Mount to the southward, although several vessels have been captured, still some cargoes of slaves have been carried off, even very recently; and we regret to state that the traffic seems by no means on the decline in those localities, although a partial check was given to it in the early part of the year, by the destruction of the slave factories of "Luis" and "Ximenes," at Seabar and Gallinas.

Theodore Canot of Cape Mount, has of late been one of the most active and successful slave factors in the neighbourhood, This man and the no less wellknown Don Luis (Lemaignere) actually visited Sierra Leone in August last in the American schooner "Patuxent," a vessel which has since been seized by a United States' cruizer.

The American squadron has of late exerted some vigilance, having captured the schooners "Merchant," and "Spitfire;" and the disposition to co-operate with our cruizers, shown in the case of the latter vessel, taken by the boats of the United States sloop of war "Truxton," in company with those of Her Majesty's steamer "Ardent," cannot but tend, if generally acted upon, to break up that systematic assistance rendered to the Slave Trade by American citizens, and which has been carried to an unusual extent during 1845.

In January Her Majesty's steam-frigate "Penelope" found three empty American schooners concealed in the creeks of the Rio Pongas, under very suspicious circumstances. In the same month an American vessel called the "Atalanta," was transferred at Cape Mount, where a cargo of slaves was immediately shipped; the American crew quitting the "Atalanta" on one side, as the slaves were sent up the other. The "Atala" and some other vessels condemned in these courts, are supposed to have been delivered by the Americans in a similar manner, and the practice is notorious. 
A great blow has been struck at the Slave Trade, especially that carried on between Lagos and Bahia, by the very numerous seizures in the Bight of Benin, yet even from that part of the coast, despite the utmost exertions of the squadron, some cargoes of slaves have been carried off.

There has also been, notwithstanding the active endeavours of the Portuguese Government to suppress it, a very extensive traffic in the neighbourhood of the Portuguese possessions to the southward of the Line. Our own list of captures made in that direction is considerable; and it appears from the following extract of a letter addressed to Commodore Jones, by Her Majesty's Arbitrator at Loanda, dated the 12th of August last, that several cases have been brought before the Mixed Court established in that settlement:

"During the three months I have resided here, I have received positive intelligence of eleven slave vessels which have appeared on the coast, of which number seven have been captured or destroyed by the Portuguese squadron, two by British cruizers, and two have escaped with cargoes of slaves; all within a distance of fifty miles on either side of the city of Loanda."

In our report for last year we commented on the apparent revival of the Cuba Slave Trade; the result of the proceedings of 1845 would however induce us to hope that that revival was merely partial and. temporary; the number of Spanish slave vessels shown in the present Return being considerably less than in that of 1844, and all, it will be seen, were captured within the first five months of the year. One third of them were employed in carrying slaves to Brazil; so that in fact, not more than six of the nine Spanish vessels detained, were engaged in Spanish Slave Trade.

On the other hand, the number of Brazilian slave vessels captured during 1845 , has far exceeded those detained in any previous year, notwithstanding that the British and Brazilian Court of Mixed Commissions ceased to adjudicate in August last, and that with the exception of the "Adelaide," detained on the I2th of August, and subsequently given up, no vessel furnished with Brazilian papers was taken by any of Her Majesty's cruizers, between the 23rd of July and the 22 nd of October, in consequence of the expiration of the Convention of 1817, and the Act 8 and 9 Victoria, cap. 122, not having come into operation,

This increase will be more evident from the following general statement of cases of Brazilian slave vessels, and of vessels engaged in Brazilian Slave Trade, brought here for adjudication during the year 1845:

Brazilian vessels proceeded against in the British and Brazilian Court of Mixed Commission at Sierra Leone, during the year $1845 ; 21$

Brazilian vessels captured between the 25th of April and 13th of August, and brought to Sierra Leone but not proceeded against, there being no court competent to take cognizance of the cases; $4{ }^{11}$ 
Brazilian vessels captured under the Act 8 and 9 Victoria, cap. 122, and proceeded against in the Vice-Admiralty Court at Sierra Leone; 52)

Vessels engaged in Brazilian Slave Trade, but without papers or other evidence to prove a national character, prosecuted and condemned in the ViceAdmiralty Court at Sierra Leone, during the year 1845, and under the Act 2nd and 3rd Victoria, cap. 73; 10

Spanish vessels engaged in Brazilian Slave Trade, condemned in the British and Spanish Mixed Court of Justice at Sierra Leone, during 1845: 3

Total: 43

1) These were the "Princeza," "Emprehendedor," "Quatro de Março," and "Adelaide," reported in despatches marked Brazil, of the 21st and 29th of August and 16th of September last. The "Emprehendedor" was broken up here, being unseaworthy; the "Princeza" was subsequently re-captured whilst engaged in a fresh slaving expedition, and was condemned in the Vice-Admiralty Court. The others are said to have returned to the Brazils.

2) On one of these, the "Boa Sorte," sentence has not yet been passed.

From this it will be seen that forty-three cases of Brazilian Slave Trade were brought for adjudication into Sierra Leone alone; a fact from which some idea may be formed of the great extent of the traffic as regards Brazil, especially if there be taken into consideration the cases of Brazilian Slave Trade sent into St. Paul de Loanda, and to the Vice-Admiralty Court at St. Helena, by Her Majesty's cruizers during the past year.

These are stated to have been eighteen in number, which would give a total of sixty-one cases of Brazilian Slave Trade during 1845, without including the numerous captures made by the Portuguese squadron, or by Her Majesty's cruizers attached to the Cape of Good Hope and employed on the East Coast, where the Brazilian Slave Trade is at present said to be in great activity; but we have no sufficient information respecting the proceedings of either of those naval forces to enable us to include them in our calculation.

We have it however in our power, through the kindness of Commodore Jones, to afford a proximate view of the proceedings of the squadron under his command, and cruizing on the West Coast, between the latitudes of $20^{\circ}$ North, and $15^{\circ} 54^{\prime}$ South.

From a list which the Commodore has furnished to us, it appears that the whole number of slave vessels detained or destroyed on this coast by Her Majesty's cruizers, between the 1st of January and the 9th instant, is seventy-five. (101 slave vessels altogether were captured or destroyed by the squadron between the 1st of April, 1844, and 9th of December, 1845.)

Of these the nationality, as shown by the colours and papers were, thirtyseven Brazilian, ten Spanish, two Portuguese, two Sardinian, one American the schooner "Merchant," reported in Despatch, marked Spain, of the 1 st of 
November. This vessel was given up, but was recaptured off Sierra Leone by the American Commodore on the 29th of November), one Monrovian, and twentytwo without colours or papers, and the cases were sent for disposal as follows:

- Sierra Leone 55* / St Helena 16 / Loanda 2 / Genoa 2

*Some of these have not arrived at Sierra Leone, and it seems probable have gone to St. Helena

A result so highly creditable to the vigilance and activity of Her Majesty's cruizers, speaks much also for the excellent and judicious arrangements of Commodore Jones, aud for the general efficiency of the force under his command.

So destructive to the traffic has the employment of steam vessels of war been found, that the Brazilian dealers have been driven to the desperate shift of fitting out steam slavers. One of these, the "Cacique," has been captured, but another, we regret to learn, has recently got off safely from the Congo with a cargo of seventeen hundred negroes.

The "Cacique" was built in New York, where she was called the "Tigress," and ran for a short time as a passage boat worked by a screw propeller. In March last she was purchased by a Brazilian named Seixas, and taken, it is said, under American colours to Pernambuco, where paddle-wheels were fitted to her and the machinery of her engines removed to the deck, so as to leave an open hold fore and aft; a slaving equipment was added; the crew, with the exception of the four American engineers, changed; a long gun and small arms for thirty hands put on board; and in August she started for Cabinda, off which she arrived on the 20th of September.

At Cabinda Mr. Seixas, the owner, went on shore, and found one thousand slaves awaiting him really for shipment, but fifteen hundred being the steamer's complement, he ordered her to sea for ten days until the deficiency should be collected. She was, however, fortunately fallen in with and detained on the 26th, by Her Majesty's steam frigate "Penelope," having previously easily evaded Her Majesty's sloop "Cygnet" and another cruizer, by steering head to wind.

Some of the slave vessels captured this year, were furnished with the flags of Portugal, America, France, and Buenos Ayres, in addition to the colours under which they professed to sail; and some had equipments of an unusually piratical character, being heavily armed, and provided with crews of great numerical strength, and composed for the most part of persons of different nations.

The Spanish feluccas "Huracan" and "Pepito," and the Brazilian brig "Isabel," were especially remarkable in this respect; the two feluccas had long avoided capture and been successfully engaged in the traffic. We may also notice another piratical slaver, the "Borbolita," taken by the boats of Her Majesty's sloop "Pantaloon," after a very desperate defence, in which several lives were lost. This prize was condemned in the Vice-Admiralty Court. 
An instance also of singular ferocity was exhibited by the Brazilian crew of another vessel, the slave-laden brigantine "Echo," detained by the boats of Her Majesty's sloop "Wasp," shortly after they had taken a Brazilian schooner named the "Felicidade," which was equipped only for the traffic. The crew of the "Echo" being excessive, had been divided and part sent to the "Felicidade," where, seizing a favourable opportunity, they overpowered and murdered the British officer and seamen in charge, and then attacked the vessel with slaves, but being unsuccessful in their attempt to regain possession of her, made off with the "Felicidade."

A few days afterwards, however, these pirates were fallen in with and recaptured by Her Majesty's sloop "Star," when the chief perpetrators of the massacre were sent to England, and the "Felicidade" despatched to Sierra Leone.

But this ill-fortuned vessel never reached her destination. Having upset in a squall, the officer and men on board managed to save themselves on a raft, and after enduring the extremities of hunger and thirst for twenty days, during which several died; the survivors were at length providentially rescued by Her Majesty's sloop "Cygnet."

The termination of the British and Brazilian Mixed Commission, the only remaining court that did not exact the breaking up of condemned slave-vessels, has for the time put an end to the possibility of such vessels being re-employed in the Slave Trade; an abuse which we trust may never be revived, feeling assured that in any future treaty which may be entered into between Great Britain and Brazil, there will be a special clause providing for the destruction of slave-vessels.

In the meanwhile, however, we have to record the fact that two of the vessels adjudicated in 1845 by the Mixed Commissions, and also two adjudicated in the Court of Vice-Admiralty at Sierra Leone, had previously been condemned here as slavers. And we think it more than probable, that some of those recently condemned in the British and Brazilian Court will also be returned into the traffic, having either been purchased at auction by known slave dealers, or by others likely to dispose of them to such parties.

Her Catholic Majesty's Government have recently appointed a Judge to the British and Spanish Mixed Court of Justice, and have also established a Consulate at Sierra Leone. In carrying out the provisions of the Treaty of 1835, we are happy to say we look forward to the cordial co-operation of our Spanish colleague, who seems sincerely disposed to use his best efforts to suppress the Spanish Slave Trade.

If, too, as we are given to understand, it be the intention of Her Catholic Majesty's Government to enforce the law against the crews of all Spanish slavers which may hereafter be condemned here; such a measure, vigorously pursued, must doubtless have some beneficial effect. At the same time, as we apprehend it is not intended to extend this interference beyond those subjects of Spain who may be found on board vessels condemned in the Mixed Court of Justice, it 
seems clear that the chief result as regards the Spanish slaver, will eventually be the destruction of papers, and other evidences of nationality.

This practice has indeed already been largely resorted to, from a desire to avoid prosecution in any of the Mixed Courts, on account of the nature of the examinations to which witnesses are subjected, and the exposure consequent upon the publication of the evidence, both of which the slavers are well aware they may escape in the Vice-Admiralty Courts, where they are not examined at all, unless the cases be contested, and even then it has not been customary hitherto for the evidence to be printed.

In concluding this report we regret to have to remark that there appears to be some ground for apprehension that the new French settlements on the coast, may tend to encourage the Slave Trade, if they be not kept under stricter surveillance than seems to be at present exercised over them.

We find that Bahia slavers are now despatched and regularly cleared for the Gaboon, of which river the French claim the sovereignty; and it is alleged that the small French military party there has afforded its protection to vessels engaged in the traffic.

As this is a subject, however, on which Commodore Jones has already, we believe, addressed the Lords of the Admiralty, it will be unnecessary for us to allude to it further.

We have, (Signed)

M.L. Melville / James Hook

Fuente: http://home.wxs.nl/ pdavis/ (Página Web dirigida por Peter Davis Zeist). 